\title{
Psychological Counseling for Alcohol Misuse in University Students
}

\author{
Zhiyu Liu ${ }^{1,2}$ \\ ${ }^{1}$ University Students' Psychological Counseling Centre, Jilin Agricultural University, Changchun, \\ 130118, China \\ ${ }^{2}$ School of Philosophy and Society, Jilin University, Changchun, 130012, China \\ 632830067@qq.com
}

Keywords: alcohol; counseling; client-centered approach; misuse; effectiveness.

\begin{abstract}
The aim of this paper is to solve or alleviate the binge drinking problem of university students from a psychological point of view. Traditionally, people were used to accepting medical interventions to alleviate their problems. But in this paper, the author considered the effects alcohol misuse makes on the university students, examined how psychological counseling helps with alcohol misused adolescents, explained the best approach, and evaluated how effective psychological counseling is for such young drinkers. The results demonstrate that the alcohol-misuse problem of university students can make a series of negative effects on individuals, groups and the society, and it can be alleviated through counseling approaches effectively. Generally, the client-centered approach is the most effective method for counseling young drinkers, while the solution-focus approach and cognitive-behavior therapy are also helpful. It is recommended that further research could focus on the application of a combination of different counseling approaches for university students.
\end{abstract}

\section{Introduction}

At the beginning of the twenty-first century, alcohol use becomes a common character of population's lives. A great number of university students enjoy beer or wine with friends on spare time for relaxation or releasing emotions. However, for many university students, they do not stop drinking at that moment, but drinking a large amount or more frequently. That is an alcohol-misuse phenomenon. Usually, such binge drinking is defined as "a daily alcohol intake that is more than twice the recommended daily safe drinking limits" [1]. For instance, a male consumes 5 or more drinks and a female has 4 or more drinks within 2 hours. Under this circumstance, they are overusing alcohol [2].

Over the previous years, many methods for alcohol related problems have been sought, according to the general clinical view which diseases can be cured by a particular treatment. Nonetheless, alcohol misuse is not a simple disease or an emotional behavior. Heather and Robertson [3] stated that problem drinking should be deemed as a learned behavioral disorder, rather than a disease. So it is agreed that there is no single treatment for this problem. But some endeavors have been implemented, such as DAATs (Drug and Alcohol Action Teams), which provides services and interventions like screening and assessment particularly for young drinkers [1]. However, the most popular and effective solution for alcohol problems is counseling as a whole.

The aim of this essay is to examine psychological counseling for university students with the alcohol-misuse problem. Firstly, it will focus on the effects of misusing alcohol on individual, group and society; secondly, it will outline the way counseling helps with alcohol misused problem; and finally, it will analyze the effectiveness psychological counseling has made in this area.

\section{The Effect of Alcohol Misuse}

Peluso and Blay [4] pointed out that alcohol use is related to a wide range of physical, mental, and social harms. Generally speaking, alcohol misuse can make a variety of negative effects on individuals, groups and the society. 
Firstly, one of the most serious outcomes alcohol use causes is physical diseases on the individual. It has been proved scientifically that once adolescents addict to alcohol use, increasing physical diseases will occur. To illustrate, liver disease, stomach ulcers, oesophageal varices, heart disease and high blood pressure, cancers, pancreatitis, damage to nerves, diabetes, sexual and menstrual impairment, brain damages including alcohol dementia, Wernicke's encephalopathy and Korsakoff's psychosis [3]. Some of those adolescent drinkers do not think their physical diseases are caused by alcohol misuse, but there is clear evidence that majority of patients with lung cancer share the feature of binge drinking in the past.

Another health risk to individual is mood. Misusing alcohol may lead to low mood, anxiety, depression or aggressive behavior. For heavy drinkers, they may even lose memory temporally. What is worse, when these negative moods appear, adolescents tend to drink more to hide their distress. As a consequence, a terrible circle formulates, which affects individuals' health seriously.

Secondly, drinking problem also makes bad effects on groups. On the one hand, families are affected. For example, once a son of a family drinks too much, he may pay less attention to his parents and other family members; more importantly, his character may turn bad. Furthermore, his parents and other family members may complaint and angry with him, or family abuse is caused in that case. On the other hand, alcohol misuse also affects the friendship between students in the university, since alcohol's effect on drinkers' mood and temper.

Thirdly, drinking problem influences the society in two ways. For one thing, as far as negative social outcomes are concerned, alcohol related deaths are responsible for $3.2 \%$ mortality [5], and it is proposed that $65 \%$ of suicide attempts and $40 \%$ violence incidents are linked to heavy drinking, respectively [3]. For another, from the financial point of view, alcohol misuse costs a great deal of money. For instance, National Health Service (NHS) pays 150 million pounds to solve alcohol problems each year. Besides that, 50 million pounds disappear per year due to alcohol related traffic accidents [3].

\section{Appropriate Approaches to Psychological Counseling}

Among the existing literatures, the most popular approach to counseling alcohol related problems is "client-centered approach". Compared with other therapies, it is a unique one owing to the fact that it is a client controlled one, rather an expert led one. Therapist in this approach focuses on the quality of the therapeutic relationship and serves as a model of a human being struggling toward greater realness [6]. In fact, the client-centered approach is based on concepts from humanistic psychology, many of which were articulated by Rogers in the early 1940s. Its basic assumptions are that clients are critically trustworthy, and they have a great deal of potential for understanding themselves and solving their own problems without therapists' direct interventions, and they are able to direct themselves if a therapeutic relationship is built [7].

Murgatroyd [8] summarizes client-centered position as: a person who needs help comes to the therapist; counselor has to understand what the client thinks and feels to help him or her properly; experts must accept everything about the client, including what they are, what they have done or have not done, what decisions they make regardless of own feelings; under the circumstance of being accepted and understood, clients are able to open themselves to the possibility of change and development. That is to say, clients themselves are responsible for setting purposes and goals for the whole procedure of therapies, and they may change their purposes and goals in different periods of the therapy within the context of a satisfied therapeutic relationship. So the client-centered approach is an experiential-oriented one, rather than a goal-oriented one.

As regards for the alcohol problem, the causes of misusing alcohol should be considered before applying specific counseling approach. Usually, binge adolescent drinkers are under negative emotions, which include high level of stress, unsatisfied interpersonal relationship and truancy. All those make a bad effect on students living on the edge of the society and have to seek beer or wine to release their distress. Bryant-Jefferies [3] concluded that the client-centered approach offer an opportunity for the clients to experience living in the centre of life. He said that, clients who had reported they always drank a lot responded "yes, that is me, always on the edge. I have never been 
in the middle". So in the process of the client-centered approach, desensitization is required for clients to start to reject "risky-edge behavior" as their main source of feeling alive, and it makes clients accept new, self-chosen middle-ground activities, interests, behaviors and other fulfilling experiences. And at last, clients can develop a new self-concept which contributes a fresh pattern of normal behavior and make them acceptable and satisfied.

Alternatively, the client-centered approach can combine with the cycle of change model to counsel those binge young drinkers. It is well known that the cycle of change model is divided into five stages: pre-contemplation, contemplation, action, maintenance and relapse. Bryant-Jefferies [3] believed client-centered approach accommodated within the framework of the cycle of change. Because the model does not tell clients what must do or how to do in each stage, but indicate what is relevant or helpful. When the counselor has empathic rapport with the inner world of the client, their motivations, hopes, fears and goals, a companion is formed. Then the communication with the counselor and the experience of the client are the key conditions to enable clients not to relapse but stick consistently. Naturally, the clients' ability of empowerment is increasing during the process of counseling, and they still tend to ask for suggestions from counselors. However, as a counselor, one can only express feelings of concern in a supportive and warm manner. That means, clients still need to make choices by themselves and change their alcohol misuse behavior in their own styles.

\section{The Effectiveness of Psychological Counseling for Alcohol Misuse}

In fact, the reason why psychological counseling is effective for university students' alcohol problem is that it owns many strong points. Firstly, young clients feel comfortable under the circumstance of client-counselor relationship. Professionals usually try their best to build a therapeutic relationship between clients and themselves, because such relationship enables clients to trust their counselors and participate actively in the activity of changing alcohol use behavior and stick on it confidently.

Secondly, supervision plays a critical role in counseling outcomes. Usually, those young drinkers do realize they need to make a change and no longer misuse alcohol; nonetheless they fail to reduce or stop drinking randomly. Consequently, it is necessary to have a supervisor with experience, although supervisors do not give direct information about how to do or what to do in some counseling approaches. The main effectiveness of supervision in counseling is helpful to rebuild the congruence, which is able to avoid sets of feelings or images arising by continuous alcohol misuse [3].

Thirdly, counseling breaks through the chemical treatments as a brief form. Traditionally, people were used to accepting medical interventions to alleviate their problems. Nevertheless, young people are all in their own individual ways, creatures of habit. They all make choices and choose activities and interests that make them feel alive, more real, able to cope, less uncomfortable. Now that adolescents own such characters, changing inappropriate alcohol habit in their own ways through counseling is the most suitable method, rather than those traditional medical treatments.

However, counseling still has some shortcomings when applying in alcohol misuse area regardless of its effectiveness. For instance, counseling is not the most perfect choice from a financial point of view. After all, a large amount of money can be spent, if counseling a great number of young drinkers.

Actually, other negative aspects which do harm in effective counseling also exist. For example, it is easy to give advice or plans when professionals feel that it is difficult for client to find out right concept or the client make an inappropriate decision. As a result, counselors have taken part in the approach without attention, which disturbs the effectiveness of the counseling approach.

Likewise, it is hard to build a therapeutic relationship which is mainly determined by the therapist's genuineness. No matter in the beginning of the approach or in the process of it, the issue of clients' trust affects the effectiveness of counseling. If counselors submerge their unique identity and style in a passive and non-directive way, they may not be harming many clients, but they may not be powerfully affecting clients [7].

What is more, since the client-centered approach is led by clients themselves, misunderstanding of the basic concepts of the approach will hide the effectiveness. Naturally, each of the actions of 
clients is made by themselves and counselors will not indicate their inappropriate step at that moment, as a consequence, they might rectify their understandings after a long period. From this point of view, counseling is a time-consuming approach for some drinkers [7].

In addition, how to deal with sensitivity to emotion becomes a challenge for counseling. For alcohol dependence users, their mood and emotion are linked to alcohol, their sensitivity would relapse the present counseling effects in a long term if their negative emotions still exist.

Finally, another problem, which decreases the effectiveness of counseling, is whether there is a most appropriate approach for alcohol misuse issue. After all there are still some critics who claim solution-focus approach and cognitive-behavior therapy are more suitable. If using an inappropriate approach to young drinkers, the effectiveness of counseling is affected inevitably.

\section{Conclusion}

To sum up, the alcohol-misuse problem of university students can make a series of negative effects on individuals, groups and the society, and it can be alleviated through counseling approaches effectively. Alcohol-misuse does harm in university students' body health and affect their normal moods on the individual level; it disturbs the harmony of those adolescent drinkers' families and interrupts the relationship between friends or classmates on the group level; meanwhile, it can bring many social issues, including traffic accidents and violence on the society level. Increasing studies about alcohol misuse provide adequate evidence to prove the effectiveness of counseling in this area. And it is believed the client-centered approach is the most effective method for counseling young drinkers, at the same time, the solution-focus approach and cognitive-behavior therapy are also helpful.

As regards for some inevitable drawbacks psychological counseling owns, it is recommended that further research could focus on the application of a combination of different counseling approaches, in order to provide a more appropriate way for counseling young drinkers in the university. Especially, compared with some other health problems, like obesity, the study on alcohol misuse is not deep or thorough enough. So there is a long way to go to make counseling more useful on helping alcohol misused university students.

\section{References}

[1]. Bryant-Jefferies, R, Counselling Young Binge Drinkers: Person-centred Dialogues. Abingdon: Radcliffe Publishing Ltd, 2006.

[2]. Sharma, M, "Binge Drinking Interventions among College Students," Journal of Alcohol \& Drug Education. US, vol. 52(2), pp.3-8, 2008.

[3]. Bryant-Jefferies, R, Counselling the Person Beyond the Alcohol Problem. London: Jessica Kingsley Publishers, 2001.

[4]. Peluso, E.D.T.P., \& Blay, S.L, "How should Alcohol Dependence be Treated? The Public View,” Alcohol \& Alcoholism. Oxford, vol, 43(5), 600-605, 2008.

[5]. Kim, J.H., Lee, S., Chow, J., Lau, J., Tsang, A., Choi, J., \& Griffiths, S.M., et al, "Prevenlance and the Factors Associated with Binge Drinking, Alcohol Abuse and Alcohol Dependence: A Population-Based Study of Chinese Adults in Hong Kong,” Alcohol \& Alcoholism, Oxford, vol, 43(3), 360-370, 2008.

[6]. Mearns, D., \& Thorne, B, Person-Centred Counselling in Action, 2nd ed., London: SAGE Publications Ltd, 1999.

[7]. Corey, G, Theory and Practice of Counselling and Psychotherapy, 7th ed., London: Thomson Learning, Inc, 2005. 
[8]. Burnard, P, Counselling Skills for Health Professionals, 4th ed., Cheltenham: Nelson Thornes Ltd, 2005. 